\title{
LAS BARRERAS FUNCIONALES Y SU INCIDENCIA EN LA OFERTA EXPORTABLE DE LAS MYPES DEL SECTOR TEXTIL CONFECCIONES
}

\author{
FUNCTIONAL BARRIERS AND THEIR INCIDENCE IN THE EXPORTABLE SUPPLY OF THE \\ MICRO AND SMALL ENTERPRISE (MSE) IN THE TEXTILE AND CLOTHING SECTOR
}

\author{
Roberto Macha Huamán \\ Universidad Nacional Mayor de San Marcos \\ Lima, Perú
}

Correo electrónico: robertomachahm@gmail.com

\section{RESUMEN}

Objetivo: Determinar de qué manera las barreras funcionales que enfrentan las Mypes del sector Textil confecciones, influyen en la oferta exportable. Las barreras funcionales que se identificaron son: Recursos Humanos, Producción y Finanzas. Método: La presente investigación está definida bajo el enfoque cuantitativo, tipo descriptivo y correlacional, diseño no experimental. Resultados: La población y muestra es no probabilística o dirigida, está conformada por catorce Mypes del sector Textil confecciones, que hayan exportado entre los años 2006-2016, habiéndose encontrado en el proceso de internacionalización de manera regular por un periodo no menor a cinco años. Conclusiones: Las deficiencias en las áreas de recursos humanos, producción y finanzas, generan limitaciones en el 50\% de las Mypes exportadoras, convirtiéndose en barreras funcionales que afectan a la oferta exportable; mientras que el $50 \%$ restante, cuenta con pocas limitaciones superando las barreras funcionales y obteniendo mejores resultados en su oferta exportable. En tal sentido, de acuerdo al coeficiente de correlación Rho de Spearman que analiza la correlación y el nivel de significancia entre las dos variables, se demuestra que las barreras funcionales que enfrentan las Mypes exportadoras del sector Textil confecciones, influyen negativamente en la oferta exportable.

Palabras clave: Barreras funcionales, oferta exportable, internacionalización.

\begin{abstract}
Objective: To determine how the functional barriers faced by MSE in the textile and clothing sector influence the exportable supply. The functional barriers that were identified are: Human Resources, Production and Finance. Method: The present investigation is defined under the quantitative approach, descriptive and correlational type and non-experimental design. Results: The population and sample is not probabilistic or directed, it is conformed by fourteen (MSE) from the textile sector, which have exported between the years 2006-2016 and have been in the process of internationalization on a regular basis for a period of not less than five years. Conclusions: The deficiencies in the areas of human resources, production and finances, generate limitations in $50 \%$ of the export (MSE) becoming functional barriers that affect the exportable supply while the remaining $50 \%$, has few limitations overcoming functional barriers and obtaining better results in its exportable offer. In this sense, according to Spearman's Rho correlation coefficient, which analyzes the correlation and the level of significance between the two variables, it is established that the functional barriers faced by the(MSE) exporters of the textile and clothing sector have a negative influence on the exportable supply.
\end{abstract}

Keywords: Functional barriers, exportable offer and internationalization. 


\section{INTRODUCCIÓN}

De acuerdo al análisis realizado a las exportaciones del sector Textil 2006-2016, registrado por la Superintendencia Nacional de Aduanas y de Administración Tributaria (SUNAT), el porcentaje de participación del sector Textil con respecto al total de las exportaciones peruanas, disminuyó notablemente de 6,2\% en 2006 a 3,3\% en 2016. Asimismo, con respecto a las exportaciones no tradicionales pasó de 27,9\% en 2006 a 11,1\% en 2016. Cabe destacar que siendo el sector Textil uno de los más importantes de las exportaciones no tradicionales, ha perdido participación y competitividad en el mercado internacional en estos últimos 11 años.

Las exportaciones del sector Textil están conformadas por prendas de vestir y confecciones, tejidos y fibras textiles. Asimismo, la participación de las exportaciones de las prendas de vestir y confecciones con respecto a las exportaciones del sector Textil disminuyó notablemente de $82,1 \%$ en 2006 a 72,9\% en 2016. Cabe destacar que, de todo el sector Textil, las prendas de vestir y confecciones, son las más afectadas porque en este periodo tuvo una reducción de 9,2\% en su participación. En tal sentido, la investigación se enfoca en la problemática de las exportaciones de las prendas de vestir y confecciones, que se ha denominado como el sector Textil confecciones.

De acuerdo al Plan Estratégico Nacional Exportador (PENX, 2003-2013), mencionados en el Plan Estratégico Nacional Exportador (PENX, 2025), del Ministerio de Comercio Exterior y Turismo (MINCETUR), se obtuvo el siguiente logro: "Desarrollo de la oferta exportable con más de 90 productos a nivel nacional, dentro de ellos se encuentra el sector Textil, fueron priorizados, brindando soporte y asistencia técnica directa a asociaciones vinculadas a estas cadenas en diferentes regiones del país". (PENX, 2025, p.43). Sin embargo, las estadísticas nos muestran que las exportaciones del sector Textil confecciones ha perdido participación y competitividad en el mercado internacional, dado que el año 2006 se exportó US\$ 1209000000 y el año 2016 sólo se exportó US\$ 871 000 000, con una reducción de $27,9 \%$ en el valor exportado. Esto nos muestra claramente que falta mucho por desarrollar la oferta exportable de este sector.

Si bien es cierto, la vigencia de los Tratados de Libre Comercio empezó el año 2009 con el Acuerdo de Promoción Comercial Perú-EE. UU y de acuerdo a MINCETUR, se han firmado 17 Acuerdos Comerciales o Tra- tados de Libre Comercio. Sin embargo, se observa que nuestra oferta exportable se encuentra poco diversificada, porque el año 2006 nuestros principales 10 países de destino representaban el $87 \%$ de las exportaciones del sector Textil, los cuales estaban conformados por Estados Unidos (59\%), Venezuela (12\%), Chile (3\%), Colombia (3\%), Italia (2\%), Ecuador (2\%), España (2\%), Francia (2\%), México (1\%), y Reino Unido (1\%) y el año 2016 nuestros principales 10 países de destino representaron el $81 \%$ de las exportaciones del sector Textil, los cuales estaban conformados por Estados Unidos (51\%), Colombia (5\%), Chile (4\%), Ecuador (4\%), Brasil (4\%), Alemania (3\%), Italia (3\%), Bolivia (3\%), Argentina (2\%), y México (2\%).

Cabe resaltar que, en estos 11 años, Estados Unidos sigue siendo nuestro principal comprador con más del $50 \%$ y de los 10 países de destino del año 2006, 06 países continúan estando dentro de los 10 principales países de destino el año 2016. Por tanto, estos Tratados de Libre Comercio y Acuerdos Comerciales suscritos por el Perú no han ayudado a diversificar mercados para el sector textil confecciones.

De acuerdo a la revista institucional de la Sociedad Nacional de Industrias (SNI, 2012), "El sector Textil-Confecciones es por excelencia una de las actividades industriales que se mueve al ritmo de la situación económica mundial y nacional" (p.12). En tal sentido, el sector debería contar con un plan de contingencia para menguar el impacto de la crisis económica mundial.

El estrato empresarial, está determinado de acuerdo a la Ley $\mathrm{N}^{\circ} 30056$, publicada el 2 de julio 2013. Asimismo, cabe precisar que la investigación estará enfocada en las Micro y Pequeñas empresas (MYPE), que en su conjunto representa 99,3\% del total de empresas en el Perú, de acuerdo al Anuario Estadístico Industrial, Mipyme y Comercio Interno del año 2016, del Ministerio de la Producción. PRODUCE. (2016). Ministerio de la Producción. Anuario Estadístico Industrial, Mipyme y Comercio Interno.

Considerando que las Mypes en el Perú representan el 99,3\% y su importancia en el desarrollo del país, según Tello (2014) "uno de los grandes problemas que padecen las Mypes es el acceso al crédito. El financiamiento es importante para fortalecer el crecimiento y desarrollo de las microempresas" (p. 212).

Por otro lado, considerando la problemática descrita 
del sector Textil confecciones, se hizo una encuesta a 28 Mypes de Lima Metropolitana, con la finalidad de recoger información primaria y enfatizar el problema referente a las barreras funcionales que enfrentan las Mypes. La respuesta de más del 50\% de las Mypes fue que no cuentan con personal capacitado para exportaciones, desconocen oportunidades comerciales en el extranjero, dificultades en el idioma inglés, poca capacidad de producción y poco capital de trabajo para financiar sus exportaciones. Cabe precisar que 12 Mypes registran actividad del Comercio Exterior y 16 Mypes no registran actividad del Comercio Exterior, pero con intenciones de exportar.

Según Escandón, Ayala y Caicedo (2013):

En su artículo científico influencia de las barreras a la exportación sobre el compromiso exportador y su incidencia en los resultados internacionales, concluye que existe un vínculo negativo y significativo entre las barreras percibidas y el compromiso exportador y el cual repercute negativamente sobre los resultados internacionales que puede obtener la empresa. Asimismo, que la presencia de barreras a la exportación, pueden ser nocivas en la posibilidad de sobrevivencia empresarial, pero puede reducirse con una mejor gestión empresarial o compromiso exportador y también señala que los resultados obtenidos deberían incentivar a los empresarios a hacer seguimiento e invertir en mejorar sus recursos financieros, humanos y gerenciales asignados a la actividad exportadora, como forma de hacer frente a las barreras de exportación y que pueden repercutir negativamente su desempeño en el mercado internacional. (p. 50).

Teniendo en cuenta que las barreras a la exportación son limitaciones que tienen las Mypes en sus diferentes áreas, los autores señalan que para superar las barreras a la exportación y obtener mejores resultados internacionales, las empresas deben invertir en recursos humanos, financieros y gerenciales.

Según Malca y Rubio (2014):

En su investigación "Obstáculo a la actividad exportadora: Encuesta a las empresas exportadoras del Perú", concluye que los resultados obtenidos en el presente estudio, son una referencia a tener en cuenta para revisar la política de promoción de exportaciones, más aún tratándose de empresas esporádicas las mismas que de contar con apoyo ad-hoc podrían mejorar su desem- peño y convertirse en exportadoras, contribuyendo de esta manera a incrementar el número de empresas exportadoras. Por otro lado, los resultados encontrados proporcionan a las empresas pautas a tener en cuenta para mejorar sus capacidades con el objeto de orientarse hacia los mercados internacionales. (p. 49).

Considerando que el proceso de internacionalización inicia con la exportación y observando el comportamiento de las Mypes peruanas en su actividad exportadora, muchas de ellas sólo se quedan en su primera exportación o exportan esporádicamente, esto se debe principalmente por limitaciones internas de la empresa y desconocimiento de los mercados extranjeros.

Paredes (2017) señala "que los factores relevantes de competitividad influyen determinantemente en la mejora de la oferta exportable de artesanía de prendas de alpaca en el consorcio Textil Titicaca" (p.13). Estos factores de competitividad identificados por el autor son: Calidad, Productividad, Recursos Humanos y Mercado.

"Las barreras a la exportación se refiere a todas aquellas restricciones que dificultan la capacidad de la empresa para iniciar, desarrollar, o sostener negocios en los mercados extranjeros" Leonidou (2004, p. 285), estas barreras se clasifican en internas y externas. En la presente investigación, sólo se tomarán las barreras internas a la exportación, debido a que estas están relacionadas a las barreras funcionales.

"Las barreras funcionales se relacionan con las ineficiencias de las diversas funciones empresariales, como los recursos humanos, la producción y las finanzas, con respecto a la exportación" citado por Leonidou (200, p. 287). Esta categoría está relacionada con limitaciones en el tiempo gerencial, insuficiencias en el personal de exportación, capacidad de producción no disponible y escasez de capital de trabajo. Estos han sido el foco de muchos estudios, revelando un impacto generalmente moderado en el comportamiento de la exportación.

De acuerdo a Medianero (2016), "Existe consenso en definir a la productividad, en términos generales, como la relación entre productos e insumos, haciendo de este indicador una medida de la eficiencia con el cual la organización utiliza sus recursos para producir bienes finales" (p. 24). En este contexto, se refiere a la relación producción y costos de los factores de producción como son; costos de capital, costos de mano de obra y costos de factores intermedios. Asimismo, encontramos una rela- 
ción con las barreras funcionales que enfrentan las Mypes exportadoras del sector textil confecciones, debido a que los costos de mano de obra está relacionada con limitaciones en el tiempo gerencial, insuficiencias en el personal de exportación (recursos humanos), los costos de capital está relacionada con la escasez de capital de trabajo (finanzas) y los costos de factores intermedios (materiales e insumos) está relacionada con capacidad de producción no disponible (producción).

Ha quedado establecido que el Perú cuenta con la capacidad para producir cualquier tipo de prenda a gran escala. Entonces caemos en el problema de fondo: el diseño peruano. El problema es que en Perú se trabaja muy poco el diseño, y no se valora por un tema cultural. La gente cree que poner un estampado es lo único que define al diseño, cuando el proceso está en la confección, estrategia de comunicación, público objetivo, selección de insumos, etc. Patiño. (Gestión.pe, 2018).

Al respecto, tener capacidad de producción no es suficiente para ser competitivo en el mercado internacional, en tal sentido, desarrollar nuevos diseños y marcas es importante para la internacionalización de las Mypes del sector textil confecciones.

Según Fonseca (2015), "La competitividad del país es la competitividad de sus productos, o sea, la competitividad agregada de sus empresas. Pero la competitividad está sujeta a múltiples factores, unos dependientes de la misma empresa y otros no" (p. 9). En tal sentido, para que las Mypes exportadoras del sector textil confecciones sean competitivas en el mercado internacional, deben superar sus propias barreras internas que dificultan el proceso de internacionalización y respecto a los factores que no dependen de la empresa, están en las manos del gobierno, quien debe brindar facilidades de acceso a recursos y otros factores que no están al alcance de las empresas. Asimismo, "La productividad en sí misma no es un indicador de competitividad. Realmente, el elemento que se requiere para lograr la competitividad es la mejoría de la productividad" Fonseca (2015, p. 27). En tal sentido, si las Mypes quieren mejorar su productividad, deben superar sus barreras internas.

De acuerdo al Ministerio de Comercio Exterior y Turismo (Mincetur, 2013), las etapas por las que atraviesa una empresa local que empieza exportar para lograr su internacionalización son CINCO (05):
La primera etapa es la exportación ocasional, donde la empresa exporta de manera esporádica, la segunda etapa es la exportación experimental, donde la empresa decide iniciar el proceso y busca mercados a los cuales exportar, la tercera etapa es la exportación regular, donde la empresa empieza a realizar exportaciones a una base estable de clientes en el exterior, en esta etapa las empresas suelen crear un departamento de exportación, la cuarta etapa es la creación de establecimientos de filiales de venta en el extranjero y la quinta etapa es la creación de establecimiento de filiales de producción, siendo esta la etapa final del proceso de internacionalización. (Mincetur, 2013, p. 14).

Considerando que el proceso de internacionalización empieza con las exportaciones ocasionales y termina con la creación de filiales de producción en los mercados extranjeros, contar con una exportación regular significa tener oferta exportable y capacidad de producción para la exportación. En tal sentido la oferta exportable está relacionada con el proceso de internacionalización.

El tener una oferta exportable no sólo significa, como muchos piensan, que se debe contar con los volúmenes solicitados por el importador o con un producto acorde con las exigencias del mercado de destino, sino que implica mucho más. Las empresas deben contar con cuatro componentes que se encuentran interrelacionados: capacidad física, capacidad económica, capacidad financiera $y$ capacidad de gestión. (Mincetur, 2013, p. 17).

El Modelo de internacionalización planteado por Johanson y Vahlne (1977), investigadores de la Universidad de Uppsala, Suecia, en la década de 1970 hicieron observaciones empíricas comprendiendo que el proceso de internacionalización es gradual y además explican que a medida aumente el conocimiento acerca de los mercados extranjeros, existe mayor compromiso internacional. El modelo de Uppsala o Modelo Sueco, se toma como teoría explicativa de la internacionalización porque ha tenido una mayor aceptación y nace como resultado del estudio realizado por Johanson y Wiedersheim-Paul (1975) con grandes multinacionales suecas. A partir de los resultados, Johanson y Vahlne (1977), desarrollaron un modelo dinámico y explicativo del proceso de internacionalización. Asimismo, Johanson y Vahlne (2009), presentaron un modelo revisado donde agregan creación de conocimiento. "En definitiva, dicho modelo ha experimentado 
2 momentos de evolución: uno inicial, que se presentó en 1977, cuando los autores crearon originalmente el modelo, y un segundo momento se presentó en 2009, cuando los autores presentaron el modelo de Uppsala revisado" Tavares, Anzo y Estrada (2014).

"La internacionalización no es más ni menos que la búsqueda de nuevos mercados distintos al mercado local, es decir consiste en exportar e invertir en el extranjero, por tanto, existen diversos grados de internacionalización” Ortega y Espinoza (2015, p. 22). Considerando la importancia del fortalecimiento de la oferta exportable y siendo el sector Textil confecciones uno de los pilares de las exportaciones no tradicionales.

La pregunta de investigación es: ¿Las barreras funcionales que enfrentan las Mypes del sector Textil confecciones, reducen la oferta exportable?

Hipótesis: Las barreras funcionales, relacionadas a recursos humanos, producción y finanzas, que enfrentan las Mypes del sector Textil confecciones, influyen negativamente en la oferta exportable.

Objetivo: Determinar de qué manera las barreras funcionales que enfrentan las Mypes del sector Textil, reducen la oferta exportable.

\section{MATERIAL Y MÉTODOS}

Respecto al tipo y diseño de investigación, de acuerdo a Hernández, Fernández y Baptista (2014) "El enfoque cuantitativo utiliza la recolección de datos para probar la hipótesis con base en la medición numérica y el análisis estadístico, con el fin de establecer pautas de comportamiento y probar teorías" (p.4). En tal sentido, la presente investigación está definida bajo el enfoque cuantitativo, bajo este enfoque, el tipo de investigación utilizado es descriptivo y correlacional, con un diseño no experimental.

Con respecto al tipo de investigación descriptivo, según Hernández et al (2014) refiere.

“únicamente pretenden medir o recoger información de manera independiente o conjunta sobre los conceptos o las variables a las que se refieren, esto es, su objetivo no es indicar cómo se relacionan éstas" (p. 92). Con respecto al tipo de investigación correlacional, según (Hernández et al) "En este tipo de estudios, tiene como finalidad conocer la relación o grado de asociación que exista entre dos o más conceptos, categorías o variables en una muestra o contexto en particular (p. 93).

De acuerdo a Hernández et al (2014) "Un estudio no será mejor por tener una población más grande; la calidad de un trabajo investigativo estriba en delimitar claramente la población con base en el planteamiento del problema" (p. 174).

La población está conformada por 14 Mypes exportadoras del sector Textil confecciones y prendas de vestir que hayan exportado entre los años 2006 y 2016, asimismo, que hayan alcanzado el proceso de internacionalización a través de las exportaciones por un periodo no menor a CINCO (05) años. Esta población se ha tomado por conveniencia investigativa, porque son las más regulares en sus exportaciones, además sus estadísticas permiten evaluar indicadores como; tasa de crecimiento promedio (TCP), promedio de participación (PP), índice de la TCP e índice del PP, y realizar la segmentación y priorización de mercados para determinar a qué mercados están exportando y recomendar la diversificación a mercados prometedores. En tal sentido, la muestra es No Probabilística o dirigida porque el trabajo investigativo se realiza en las 14 Mypes exportadoras de la población.

Para esta investigación, se utilizó un cuestionario de preguntas que se ha construido en base a las dimensiones e indicadores de las variables del problema de investigación. Asimismo, se utilizó el Alfa de Cronbach para cuantificar el nivel de confiabilidad, porque las preguntas del cuestionario tienen más de DOS (02) opciones como respuesta, de acuerdo a la escala de Likert. Posteriormente, los resultados de la encuesta se procesaron y se realizó un análisis descriptivo de las variables con sus respectivas tablas de frecuencia y la relación entre variables. También se hizo un análisis inferencial para contrastar la hipótesis utilizando el coeficiente Rho de Spearman para medir el grado de correlación o influencia entre las DOS (02) variables. Finalmente, de acuerdo al resultado arrojado por el coeficiente Rho de Spearman, el nivel de significancia resultó menor a 0.05 , por lo tanto, se rechaza la hipótesis nula y se acepta la hipótesis alterna. Para todo el proceso se utilizó el programa estadístico SPSS.

\section{RESULTADOS}

Análisis descriptivo de la variable barreras funcionales

Se observa que, de acuerdo a la encuesta realizada, el $14,29 \%$ de las empresas exportadoras (2), son deficientes 
porque presentan mayores limitaciones en las distintas áreas de la empresa tales como; recursos humanos, producción y finanzas. El 42,86\% de las empresas exportadoras (6), son aceptables porque las áreas mencionadas cumplen su tarea, pero sin superar sus limitaciones. El $21,43 \%$ de las empresas (3) son eficientes, significa que las distintas funciones de la empresa han superado las limitaciones de los recursos y capacidades de la empresa. El 21,43\% de las empresas (3) son excelentes, porque las áreas tales como; recursos humanos, producción y finanzas realizan su trabajo de manera eficiente y sostenida en el tiempo.

Tabla 1.

Tabla de frecuencias de la variable barreras funcionales.

\begin{tabular}{lcccc}
\hline & Frecuencia & Porcentaje & $\begin{array}{c}\text { Porcentaje } \\
\text { válido }\end{array}$ & $\begin{array}{c}\text { Porcentaje } \\
\text { acumulado }\end{array}$ \\
\hline Deficiente & 2 & 14,3 & 14,3 & 14,3 \\
Aceptable & 6 & 42,9 & 42,9 & 57,1 \\
Eficiente & 3 & 21,4 & 21,4 & 78,6 \\
Excelente & 3 & 21,4 & 21,4 & 100,0 \\
\hline Total & 14 & 100,0 & 100,0 & \\
\hline
\end{tabular}

Fuente: Elaboración propia.

En tal sentido, se concluye que el 57\% de las empresas exportadoras (8), cuentan con limitaciones en las barreras funcionales porque tienen deficiencias en las áreas tales como; recursos humanos, producción y finanzas. Mientras que, el 43\% de las empresas exportadoras (6), han logrado superar las barreras funcionales.

\section{Análisis descriptivo de la variable oferta exportable}

Se observa que, de acuerdo a la encuesta realizada el $14,29 \%$ de las empresas exportadoras (2), no cuentan con los volúmenes y las exigencias solicitadas por el importador por lo que se ha denominado una oferta exportable mala. El 42,86\% de las empresas exportadoras (6), presentan una oferta exportable regular, debido a que los vo- lúmenes y las exigencias no son constantes en el tiempo. El 21,43\% de las empresas exportadoras (3), presentan una oferta exportable buena, debido a que estas empresas cuentan con volúmenes y exigencias de manera sostenible en el tiempo. Así mismo el 21,43\% de las empresas exportadoras (3), presentan una oferta exportable muy buena, debido a que las empresas cuentan con capacidad física, capacidad económica, capacidad financiera y capacidad de gestión. Finalmente se puede concluir que el 53\% de las empresas exportadoras, no tienen una oferta exportable adecuada, es decir, tienen limitaciones en su capacidad física, capacidad económica, capacidad financiera y capacidad de gestión. Los restantes $47 \%$ de las empresas exportadoras tienen una oferta exportable aceptable.

Tabla 2.

Tabla de frecuencias de la variable oferta exportable.

\begin{tabular}{lrrrr}
\hline & Frecuencia & Porcentaje & $\begin{array}{c}\text { Porcentaje } \\
\text { válido }\end{array}$ & $\begin{array}{c}\text { Porcentaje } \\
\text { acumulado }\end{array}$ \\
\hline Mala & 2 & 14,3 & 14,3 & 14,3 \\
Regular & 6 & 42,9 & 42,9 & 57,1 \\
Buena & 3 & 21,4 & 21,4 & 78,6 \\
Muy buena & 3 & 21,4 & 21,4 & 100,0 \\
Total & 14 & 100,0 & 100,0 & \\
\hline
\end{tabular}

Fuente: Elaboración propia . 
Análisis descriptivo del objetivo. Barreras funcionales $y$ oferta exportable

Se presentan los resultados de la relación entre barreras funcionales y oferta exportable; según la encuesta efectuada a las empresas exportadoras, se observa que las barreras funcionales de las empresas exportadoras son deficientes cuando oferta exportable de las empresas es mala en $7,14 \%$. También, las barreras funcionales de las empresas son aceptables cuando la oferta exportable de las empresas es regular en un $42,86 \%$. De la misma forma, las barreras funcionales de las empresas son eficientes cuando la oferta exportable de las empresas es buena en un $21,43 \%$. Asimismo, se puede observar que las barreras funcionales de las empresas son excelentes cuando la oferta exportable de las empresas es muy buena en un 21,43\%. Finalmente, se puede concluir que en $50 \%$ de las empresas exportadoras, las ineficiencias de las distintas áreas de la empresa generan limitaciones y por ende se convierten en barreras funcionales, mientras que en el $50 \%$ restante, de las empresas exportadoras cuentan con pocas limitaciones superando las barreras funcionales. Estos resultados nos permiten afirmar que las barreras funcionales que presentan las empresas exportadoras influyen negativamente en la oferta exportable.

Tabla 3.

Tabla de relación entre barreras funcionales y oferta exportable.

\begin{tabular}{|c|c|c|c|c|c|c|c|}
\hline & & & & Oferta $\epsilon$ & xportable & & Total \\
\hline & & & Mala & Regular & Buena & $\begin{array}{c}\text { Muy } \\
\text { buena }\end{array}$ & \\
\hline & & Recuento & 1 & 0 & 0 & 0 & 1 \\
\hline & & $\%$ del total & $7,1 \%$ & $0,0 \%$ & $0,0 \%$ & $0,0 \%$ & $7,1 \%$ \\
\hline & & Recuento & 1 & 6 & 0 & 0 & 7 \\
\hline Barreras & Aceptable & $\%$ del total & $7,1 \%$ & $42,9 \%$ & $0,0 \%$ & $0,0 \%$ & $50,0 \%$ \\
\hline funcionales & & Recuento & 0 & 0 & 3 & 0 & 3 \\
\hline & Eficiente & $\%$ del total & $0,0 \%$ & $0,0 \%$ & $21,4 \%$ & $0,0 \%$ & $21,4 \%$ \\
\hline & & Recuento & 0 & 0 & 0 & 3 & 3 \\
\hline & Excelente & $\%$ del total & $0,0 \%$ & $0,0 \%$ & $0,0 \%$ & $21,4 \%$ & $21,4 \%$ \\
\hline & & Recuento & 2 & 6 & 3 & 3 & 14 \\
\hline Total & & $\%$ del total & $14,3 \%$ & $42,9 \%$ & $21,4 \%$ & $21,4 \%$ & $100,0 \%$ \\
\hline
\end{tabular}

Fuente: Elaboración propia.

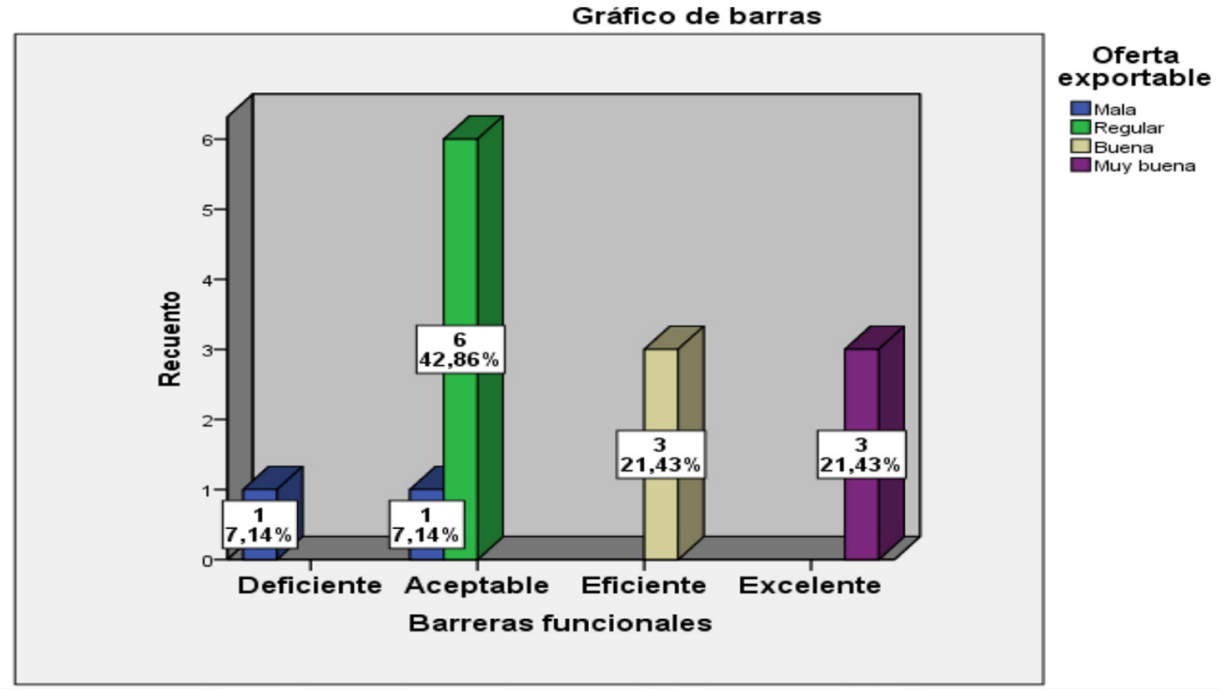

Figura 1. Relación entre barreras funcionales y oferta exportable.

Fuente: Elaboración Propia. 
Prueba de hipótesis

Para el análisis inferencial se utilizó el estadístico coeficiente de correlación de Spearman:

H0: Las barreras funcionales, relacionadas a recursos humanos, producción y finanzas, que enfrentan las Mypes del sector Textil, no influyen negativamente en la oferta exportable.

H1: Las barreras funcionales, relacionadas a recursos humanos, producción y finanzas, que enfrentan las Mypes del sector Textil, influyen negativamente en la oferta ex- portable.

El resultado del coeficiente de correlación Rho de Spearman es 0,965 ; por tanto existe una correlación positiva muy alta entre las variables barreras funcionales y oferta exportable y como el nivel de significancia resultó menor a $0,05(0,000<0,05)$ se rechaza la hipótesis nula $(\mathrm{H} 0)$ y se acepta la hipótesis alternativa $(\mathrm{H} 1)$; finalmente se cuenta con evidencia estadística para afirmar que las barreras funcionales, relacionadas a recursos humanos, producción y finanzas, que enfrentan las Mypes del sector Textil, influyen negativamente en la oferta exportable.

Tabla 4.

Tabla de correlación Rho de Spearman: prueba de hipótesis.

\begin{tabular}{|c|c|c|c|c|}
\hline & & & $\begin{array}{c}\text { Barreras } \\
\text { funcionales }\end{array}$ & $\begin{array}{c}\text { Oferta } \\
\text { exportable }\end{array}$ \\
\hline \multirow{6}{*}{$\begin{array}{l}\text { Rho de } \\
\text { Spearman }\end{array}$} & \multirow{3}{*}{$\begin{array}{l}\text { Barreras } \\
\text { funcionales }\end{array}$} & Coeficiente de correlación & 1,000 & $0,965^{* *}$ \\
\hline & & Sig. (bilateral) & & 0,000 \\
\hline & & $\mathrm{N}$ & 14 & 14 \\
\hline & \multirow{3}{*}{$\begin{array}{l}\text { Oferta } \\
\text { exportable }\end{array}$} & Coeficiente de correlación & $0,965^{\text {** }}$ & 1,000 \\
\hline & & Sig. (bilateral) & 0,000 & \\
\hline & & $\mathrm{N}$ & 14 & 14 \\
\hline
\end{tabular}

Fuente: Elaboración propia .

\section{DISCUSIÓN}

De los resultados obtenidos en la investigación, de acuerdo al coeficiente de correlación Rho de Spearman que analiza la correlación y el nivel de significancia entre las dos variables, se demuestra que las barreras funcionales que enfrentan las catorce Mypes exportadoras del sector Textil confecciones, influyen negativamente en la oferta exportable.

Se comprueba que: las deficiencias del personal no capacitado en las áreas de gerencia y exportaciones, la falta de capacidad de producción por los escasos recursos utilizados y la escasez de capital de trabajo por la poca capacidad de financiamiento, que enfrentan las Mypes del sector Textil confecciones, influyen negativamente en la oferta exportable.
En tal sentido se concluye:

Las deficiencias de las distintas funciones de la empresa como son las áreas de recursos humanos, producción y finanzas, generan limitaciones en el 50\% de las Mypes exportadoras y por ende se convierten en barreras funcionales que afectan a la oferta exportable, mientras que en el $50 \%$ restante, cuentan con pocas limitaciones superando las barreras funcionales y obteniendo mejores resultados en su oferta exportable.

Las deficiencias en el área de recursos humanos, como es el personal no capacitado en gerencia y exportaciones, generan limitaciones en el 35,7\% de las Mypes exportadoras y por ende se convierten en barreras funcionales que afectan a la oferta exportable, mientras que en el 64,3\% restante, son más eficientes y superan las barreras funcionales obteniendo una mejor oferta exportable. Asimis- 
mo, la falta de capacidad de producción por los escasos recursos utilizados genera limitaciones en el $50 \%$ de las Mypes exportadoras y por ende se convierten en barreras funcionales que afectan a la oferta exportable, mientras que en el 50\% restante, cuentan mayor capacidad de producción superando las barreras funcionales y obteniendo mejores resultados en la oferta exportable. De la misma forma, la escasez de capital de trabajo por la poca capacidad de financiamiento genera limitaciones en el 78,57\% de las Mypes exportadoras y por ende se convierten en barreras funcionales que afectan a la oferta exportable, mientras que en el $21,43 \%$ restante, han superado los problemas financieros obteniendo mejores resultados en la oferta exportable.

Finalmente, los resultados nos muestran que las barreras funcionales influyen negativamente en la oferta exportable y, por tanto, confirma lo mencionado por Vozikis y Mescon (1985) "Las barreras funcionales se relacionan con las ineficiencias de las diversas funciones empresariales, como los recursos humanos, la producción y las finanzas, con respecto a la exportación" (Leonidou, 2004, p. 287). Asimismo, Asimismo, según Escandón, Ayala \& Caicedo (2013) "la presencia de barreras a la exportación, pueden ser nocivas en la posibilidad de sobrevivencia empresarial, pero puede reducirse con una mejor gestión empresarial o compromiso exportador" y también señalan que "los resultados obtenidos deberían incentivar a los empresarios a hacer seguimiento e invertir en mejorar sus recursos financieros, humanos y gerenciales asignados a la actividad exportadora”. En tal sentido, las Mypes exportadoras del sector Textil confecciones deben invertir en estas áreas y superar estas barreras para obtener una mejor oferta exportable y un mejor desempeño en la internacionalización de la Mype peruana.

\section{REFERENCIAS BIBLIOGRÁFICAS}

Escandón, D., Ayala, A., \& Caicedo, M. (2013). Influencia de las barreras a la exportación sobre el compromiso exportador y su incidencia en los resultados internacionales. Rev. esc.adm.neg.,75 (2), 38-55.

Fonseca, R. (2015). Competitividad. La clave del éxito empresarial. Bogota, Colombia: Alfaomega colombiana S.A.

Hernández, R., Fernández, C., \& Baptista, M. (2014). Metodología de la investigación (6ta. ed.). México D.F.: McGRAW-HILL / INTERAMERICANA EDITORES.
Johanson, J., \& Vahlne, J.-E. (1977). The internationalization of the firma Model of knowledge development and increasing foreign market commitmnents. Journal of International Business Studies, 8 (1), 23-32.

Johanson, J., \& Vahlne, J.-E. (2009). The Uppsala internationalization process model revisited: From liability of foreignness to liability of outsidership. Journal of International Business Studies, 40, 1411-1421.

Johanson, J., \& Wiedersheim-Paul, F. (1975). The internationalization of the firm-four Swedish cases. Journal of Management Studies, 12 (3), 305-323.

Leonidou, L. (2004). An Analysis of the Barriers Hindering Small Business Export Development. Journal of Small Business Management, 42(3), 279-302.

Ley 30056. (2013). Leyes para facilitar, impulsar el desarrollo productivo y el crecimiento empresarial.obtenido de: http://blog.pucp.edu.pe/blog/ tributosyempresa/2013/07/03/ley-30056-que-modifica-diversas-leyes-para-facilitar-la-inversi-n-impulsar-el-desarrollo-productivo-y-el-crecimiento-empresarial/

Malca, O., \& Rubio, J. (2014). Obstáculos a la actividad exportadora: Encuesta a las empresas exportadoras del Perú. (Centro de Investigación Universidad del Pacífico-CIUP), Universidad del Pacífico, Lima.

Medianero, D. (2016). Productividad Total. Teoría y Métodos de Medición. Lima: Editorial Macro.

Mincetur. (2013). El ABC del Comercio Exterior. Guía Práctica del Exportador, III. Lima: Ministerio de Comercio Exterior y Turismo.

Ortega, A., \& Espinoza, J. (2015). Plan de Internacionalización Empresarial. Manual Práctico. Madrid: Esic Editorial.

Paredes, B. (2017). Análisis de la competitividad empresarial y su incidencia en el mercado internacional del consorcio textil Titicaca Puno, periodo 2014-2015. Universidad Nacional del Altiplano, Puno.

Patiño, M. (10 de enero 2018). Diseño de modas, el eslabón perdido que necesita la industria textil para despegar en el Perú. Gestión.pe. Obtenido de: https://gestion.pe 
PENX. (2003-2013). Plan Estratégico Nacional Exportador - PENX 2003 - 2013 .Lima:Ministerio de Comercio Exterior y Turismo. Obtenido de: https://www.mincetur. gob.pe/comercio-exterior/plan-estrategico-nacional-exportador/penx-2003-2013/

PENX. (2025). Plan Estratégico Nacional Exportador 2025. Lima:Ministerio de Comercio Exterior y Turismo. Obtenido de: https://www.mincetur.gob.pe/wp-content/ uploads/documentos/comercio_exterior/plan_exportador/Penx_2025/PENX_FINAL_101215.pdf

PRODUCE. (2016). Anuario Estadístico Industrial, Mipyme y Comercio Interno. Ministerio de la Producción. Obtenido de: http://ogeiee.produce.gob.pe/images/oee/ Anuario\%20Estad\%C3\%ADstico\%202016_2.pdf

SNI. (2012). Sociedad Nacional de Industrias. Obteni- do de: http://www2.sni.org.pe/servicios/publicaciones/ download/Industria_Peruana_872.pdf

SUNAT. (2006-2016). Superintendencia Nacional de Aduanas y de Administración Tributaria. Obtenido de: http://www.sunat.gob.pe/estad-comExt/modelo_web/ web_estadistica.htm

Tavares, S., Anzo, E., \& Estrada, L. M. (2014). La cooperación internacional en la internacionalización de pequenas y medianas empresas de Medellín: un estudio de caso comparado. Estudios Gerenciales, 30(132), 314-324.

Tello, S. (2014). Importancia de la micro, pequeñas y medianas empresas en el desarrollo del país. LEX,12(14)20. Obtenido de: file://C:/Users/PC-Alumnos/Downloads/ Dialnet-ImportanciaDeLaMicroPequenasYMedianasEmpresasEnElD-5157875.pdf 